\title{
A melhor idade: o curso de Relações Públicas da UFSM aos seus 40 anos ${ }^{1}$
}

\author{
The best age: stroke PR UFSM its $\mathbf{4 0}$ years
}

\author{
Marcos Júnior Junges Panciera \\ Universidad Federal de Santa Maria (Brasil) \\ marcos.panciera@gmail.com \\ Dr. Flavi Ferreira Lisboa Filho \\ Universidad Federal de Santa Maria (Brasil) \\ flavilisboa@gmail.com \\ Carlos Eduardo Figueirôa Arruda \\ Universidad Federal de Santa Maria (Brasil) \\ dudu figueiroa@hotmail.com
}

Recibido: 30 de julio de 2013

Aceptado y Publicado: 1 de octubre de 2013

\section{Resumen}

O resgate da trajetória histórica do curso de Comunicação Social - habilitação em Relações Públicas - RP - da Universidade Federal de Santa Maria - também grafado UFSM - já foi realizado por Silveira (2003), Barichello e Martins (2005) e Barichello et al.(2012), a bibliografia que privilegia a história do curso de RP da UFSM é bastante rica, ainda assim, esforços são necessários para complementação de aspectos não abordados. O presente trabalho pretende inter-relacionar, analisar e complementar estudos que resgatam a memória e avaliam as condições presentes do bacharelado em Relações Públicas da UFSM, a partir dos resultados do projeto de pesquisa "Uma proposta de auto-avaliação e resgate da história do curso de Relações Públicas".

O curso de Relações Públicas da UFSM completou 42 anos de existência no dia 15 de abril de 2013, data que marca oficialmente a sua criação através de decreto lei, publicado no Diário Oficial da União no ano de 1971. Durante as suas quatro décadas de experiência

\footnotetext{
${ }^{1}$ Artículo producto de una Ponencia presentada en el VI Encuentro Panamericano de Comunicación COM PANAM 2013 celebrado la Escuela de Ciencias de la Información de la Universidad Nacional de Córdoba (Argentina), durante los días 5, 6 y 7 de junio de 2013.
} 
formativa a graduação passou por diversas modificações internas: curriculares e estruturais. Em paralelo, as mudanças externas de localização, afirmação institucional, até alterações políticas, econômicas e sociais relacionadas à cidade de Santa Maria, região central do Rio Grande do Sul, e de demandas estaduais e federais.

O estudo complementa em alguns aspectos questões não presentes na bibliografia supracitada, primeiro um quadro atualizado completo de todos os dirigentes do Departamento de Ciências da Comunicação, o que auxiliará a compreender o desenvolvimento do curso; outro ponto é a trajetória das assessorias de relações públicas e suas respectivas divulgações, por meio, da Mostra de Assessorias de Relações Públicas MARP -; segundo, o momento atual do curso, como registro histórico à passagem para quinta década e sexta grade curricular. Posto que, as transformações necessitam de registro, considerando mudanças constantes e aceleradas que ocorrem na contemporaneidade.

Nesse viés, nosso objetivo é resgatar a história do curso de Relações Públicas, para que possamos refletir sobre suas perspectivas a partir do seu desenvolvimento e das diversas contribuições sociais e científicas ao longo dos seus 40 (quarenta) anos de existência. $O$ trabalho se desenrola através das quatro décadas passadas e ao fim projeta perspectivas para quinta década que se iniciou, seguindo a estrutura seguinte, (1) primeira década, criação do curso e desenvolvimento inicial; (2) segunda década, as primeiras assessorias; (3) terceira década, fortalecimento da atividade profissional; (4) quarta década, grande desenvolvimento da Comunicação Social; (5) quinta década, perspectivas futuras.

\section{Abstract}

The rescue of the historical trajectory of the Social Communication course - qualification in Public Relations - PR - Federal University of Santa Maria - also spelled UFSM - has been accomplished by Silveira (2003), Barichello and Martins (2005) and Barichello et al. (2012), the literature that focuses on the history of the course of RP UFSM is quite rich, yet efforts are needed to complement the aspects not addressed. This work intends to inter -relate, analyze and complementary studies that recover memory and evaluate the present conditions of the BA in Public Relations UFSM, from the results of the research project "A proposal for self evaluation and rescue course history Public Relations".

The course Public Relations UFSM completed 42 years of existence on April 15, 2013, a date that officially marks its creation by decree law published in the Official Gazette in 1971. During his four decades of training experience graduation went through several internal modifications: curricular and structural. In parallel, the external changes of location, institutional affirmation, changes to political, economic and social issues related to the city of Santa Maria, the central region of Rio Grande do Sul, and state and federal requirements. 
The study complements in some respects issues not present in the bibliography above, first complete an updated picture of all officers of the Department of Communication Sciences, which will help to understand the development of the course, another point is the trajectory of public affairs offices and their related disclosures through, the show Counseling Public Relations - APR - and second, the moment of course, as the historical record for the transition to fifth decade and sixth grade curriculum. Since the transformations require registration, considering constant changes and accelerating developments in contemporary times.

In this vein, our goal is to rescue the history of Public Relations course , so we can reflect on their perspectives from their development and the various social and scientific contributions throughout their forty (40) years of existence. The work unfolds through the past four decades and the projected outlook for end fifth decade that began following the following structure, (1) first decade, course creation and early development, (2) second decade, the first advisory; (3) third decade, strengthening of professional activity, (4) fourth decade, great development of social communication, (5) fifth decade, future prospects.

Palabras Clave: Formação, Educação, Eventos, Relações Públicas

Key Words: Training, Education, Events, Public Relations

\section{Introducción}

A perspectiva brasileira de Relações Públicas é estabelecida pelo Conselho Federal de Relações Públicas - CONFERP- órgão profissional máximo da área no Brasil, para o Conselho: Relações Públicas é a atividade e o esforço deliberado, planejado e contínuo para estabelecer e manter a compreensão mútua entre uma instituição pública ou privada e os grupos de pessoas a que esteja, direta ou indiretamente, relacionada. Todo cargo designado como relações-públicas deve ser assumido por um profissional com formação específica e registrado no órgão federal.

O Projeto Político Pedagógico do Curso de Relações Públicas da UFSM (2010) como currículo é aprovado pelo Ministério da Educação, e segue a diretriz proposta pelo CONFERP, formando profissionais conforme a legislação de trabalho em relações públicas. O Curso de Relações Públicas prevê então o desenvolvimento de competências para:

- Pesquisar, planejar e executar atividades e de programas de avaliação, com a finalidade de estabelecer e manter compreensão mútua e promover a integração entre as instituições e seus públicos; 
- Compreender a natureza das linguagens e dos fenômenos culturais envolvidos nos sistemas atuais de comunicação e, em especial, relativos ao processo de comunicação entre as instituições e seus públicos;

- Entender o papel dos sistemas e processos de comunicação na contemporaneidade, bem como a evolução histórica destes e suas relações com a cultura, a política e a economia;

- Interpretar a realidade social e o desenvolvimento do pensamento especulativo sobre perspectivas futuras de atuação.

Entre as áreas de atuação do profissional pode-se citar: entidades, instituições públicas e privadas; organizações não governamentais; sindicatos; agências de comunicação; cooperativas; empresas jornalísticas; assessorias de comunicação; partidos políticos e outras entidades que requeiram as atividades do profissional de relações públicas com finalidades institucionais ou em apoio às áreas de recursos humanos e de marketing.

O Curso de RP da UFSM na atualidade é composto por $122^{2}$ (cento e vinte dois) alunos, 6 (seis) professores específicos e mais 8 (oito) professores de núcleo comum, compartilhados com os outros três Cursos da Comunicação Social do Departamento de Ciências da Comunicação. Está estabelecido desde 1983 no mesmo local, prédio 21 do campus universitário. Conta com uma agência experimental, um laboratório de prática e um laboratório de opinião pública. Além dos laboratórios do núcleo comum. Desenvolve sua função social com ações de extensão aplicadas, principalmente, aos órgãos da Universidade Federal de Santa Maria através das Assessorias de Relações Públicas.

Historicamente, a graduação, criada em 1971, dentro da área de Comunicação Social da UFSM nasceu pouco após a regulamentação da profissão no Brasil, em 1969. Manteve-se sólida na formação de profissionais preparados para as demandas sociais e mercadológicas. Mesmo nos primórdios do Departamento de Ciências da Informação - hoje, Ciências da Comunicação -, em que faltavam equipamentos e materiais, e principalmente, conhecimento sobre a profissão na cidade de Santa Maria e região central do estado, ainda assim, embora houvesse adversidades, desde seu inicio, grande esforço por parte dos gestores e docentes para proporcionar uma formação privilegiada. De modo que, as adaptações curriculares do Curso seguiram uma direção social, preocupando-se com a formação e a contribuição para sociedade.

\footnotetext{
${ }^{2}$ Número referente à quantidade de alunos no mês de dezembro do ano de 2012 (dois mil e doze), obtidos junto ao SIEUFSM.
} 
(...) na tentativa de responder aos desafios impostos em cada época, alterações nas grades curriculares representam e incorporam lógicas sociais, políticas, institucionais e mercadológicas externas. Representam, também, um empenho no sentido de credenciar os egressos de Relações Públicas da UFSM com saberes e competências que deem conta das exigências sociopolíticas, teóricas e técnicas da profissão. Enfim, um esforço para sair de uma crise de legitimidade e assumir uma postura mais democrática e emancipatória como convém à universidade, entendida como um bem e um espaço público. (BARRICHELO et al. 2012, p. 207)

Fica evidente a partir da citação anterior a preocupação sempre presente em tentar adequar as proposições às demandas do mercado e da formação cidadã dos discentes do Curso.

Antes de aprofundar o desenvolvimento do curso através das décadas de existência, é importante expor o quadro de dirigentes ao longo da história do Curso. As seções Dirigentes da primeira, segunda e terceira década são informações retiradas de Barichello e Martins (2005). Dirigentes da quarta década é um conjunto de dados estruturado através de pesquisa de arquivos do Centro de Ciências Sociais e Humanas - CCSH -, portarias entre os anos de 2002 e 2012.

1. Dirigentes da primeira década:

Coordenadores

- Antônio Abelin (Coordenador 1972 - 1977)

- Luiz Carlos Flores Grassi (Coordenador 1978 - 1981)

- Paulo Roberto de Oliveira Araújo (Coordenador 1981 - 1983)

2. Dirigentes da segunda década: Coordenadores e Vice-diretores

- Paulo Roberto de Oliveira Araújo (Coordenador 1981 - 1983)

- Rosane Manica RizziCatani (Coordenadora 1984 - 1985)

- Veneza Veloso MayoraRonsini (Coordenadora 1986 - 1988)

- Maria Ivete Trevisan Fossá (Coordenadora 1988 - 1990)

- Zenir Maria ForgiariniCecchin (Vice-Diretora 1990 - 1992) 
Chefes de Departamento e Diretores

- Lenira Loureiro (Chefe do Departamento 1984 - 1986)

- Eunice Teixeira Olmedo (Chefe do Departamento 1986 - 1988)

- Adair Caetano Peruzollo (Chefe do Departamento 1988 - 1990)

- Maria Ivete Trevisan Fossá (Diretora 1990 - 1992)

3. Dirigentes da terceira década

\section{Vice-diretores e Coordenadores}

- Gisele MarchioriNussbaumer (Vice-Diretora 1993 - 1994)

- Eugenia Mariano da Rocha Barichello (Vice-Diretora 1994)

- Rogério Ferrer Koff (Vice-Diretor 1995 - 1997 e Coordenador 2002 - 2003)

- Jocélia Maris Mainardi (Coordenadora 1997 - 1998)

- Paulo Roberto Araújo (Vice Coordenador 1997 - 1998)

- JaneaKessler (Coordenadora 1999 - 2000, 2001 - 2002)

- Márcia Amaral (Vice Coordenadora 1999)

- Cristiane Pizzuti dos Santos (Coordenadora 2000 - 2001)

\section{Diretores e Chefes de Departamento}

- Ada Cristina Machado Silveira (Diretora 1993 - 1995)

- Eugenia Mariano da Rocha Barichello (Diretora 1995 - 1997 e Chefe de Departamento 1998, 2001 - 2003, 2004 - 2005)

- Rondon Martin Souza de Castro (Chefe de Departamento 1998 - 2001)

4. Dirigentes da quarta década

\section{Coordenadores}

- Rondon Martin Souza de Castro (Coordenador Pró-Tempore 2002) 
- Rogério Ferrer Koff (Coordenador 2002)

- Maria Ivete Trevisan Fossá (Coordenadora 2003 - 2004)

- Rogério Ferrer Koff (Coordenador Pró-Tempore 2004 - 2005)

- Maria Ivete Trevisan Fossá (Coordenadora 2005)

- Luciana PellinMielniczuk (Coordenadora Pró-Tempore 2006)

- Márcia Franz Amaral (Coordenadora Pró-Tempore 2006)

- Eugenia Mariano da Rocha Barichello (Coordenadora Pró-Tempore 2006 - 2007)

- Elisângela Carlosso Machado Mortari (Coordenadora 2007 - 2009, Pró-Tempore 2009)

- Rosane Rosa (Coordenadora do curso de Relações Públicas 2009 - 2010)

- Elisângela Carlosso Machado Mortari (Coordenadora Pró-Tempore do curso de Relações Públicas 2010 - 2011)

- Janderle Rabaiolli (Coordenador Pró-Tempore 2011)

- Flavi Ferreira Lisboa Filho (Coordenador do curso de Relações Públicas 2011 - 2012)

- Rejane Oliveira Pozzobon (Coordenadora Pró-Tempore do curso de Relações Públicas 2012)

\section{Coordenadores da Pós-Graduação}

- Ada Cristina Machado Silveira (Coordenadora da Pós-Graduação Pró-Tempore 2005 - 2007)

- Eugênia Maria Mariano da Rocha Barichello (Coordenadora da Pós-Graduação 2007 - 2009, 2009, 2010, 2011, 2012)

Diretores e Chefes de Departamento

- Eugênia Maria Mariano da Rocha Barichello (Chefe de Departamento 2002 - 2003)

- Veneza Veloso MayoraRonsini (Chefe de Departamento Pró-Tempore 2005) 
- Ada Cristina Machado Silveira (Chefe de Departamento 2005 - 2007, 2009 - 2010, 2011 - 2012)

- Flavi Ferreira Lisbôa Filho (Chefe de Departamento 2012 - 2013)

\section{A primeira década}

A graduação, criada em 1971, dentro da área de Comunicação Social da Universidade Federal de Santa Maria nascia pouco após a regulamentação da profissão no Brasil, em 1969, sendo o curso da UFSM um dos pioneiros no país. A formação manteve-se sólida no preparo de profissionais às demandas sociais e mercadológicas. Mesmo nos primórdios quando o departamento chamava-se Departamento de Ciências da Informação - hoje, Ciências da Comunicação -, em que faltavam equipamentos e materiais, e principalmente, conhecimento sobre a profissão na cidade de Santa Maria e região central do estado, ainda assim, embora as adversidades havia grande esforço por parte dos gestores e docentes para proporcionar uma formação privilegiada.

A primeira turma de formandos de Comunicação Social da UFSM evidência o desconhecimento da profissão, a habilitação era escolhida no quarto semestre de curso, após o término da formação básica. Em 1975 graduam-se apenas três pessoas em Relações Públicas, um número pequeno que aumentaria com o passar dos anos, e por vezes, ultrapassa os demais cursos, assim como por algumas vezes foi o curso mais procurado da Comunicação Social e o 6ำ (sexto) mais concorrido do processo seletivo da instituição de ensino por diversas ocasiões.

Assim, da primeira década podemos inferir que a qualidade de ensino sempre buscou ser um tom marcante do curso, à medida que a profissão reconhecida perante o mercado de trabalho regional e novos aparatos tecnológicos e didáticos foram adquiridos, novas ideias agregadas, a experiência aprimorou-se e o diploma ganhou peso.

\section{A segunda década}

Na década de 80 , segundo Silveira (2003) a prática profissional dentro da academia recebe maior ênfase através da criação de uma Agência Experimental, são prestadas assessorias de relações públicas para instituições externas à universidade, principalmente de caráter comunitário e público. 
As assessorias de relações públicas configuram-se como o ponto forte do contributo social do Curso, conforme Silveira (2003), o primeiro registro de assessorias na área de Relações Públicas, na UFSM, data do ano de 1983, quando foi criada uma Agência Experimental. Barichello e Martins (2005) complementam que o primeiro relato de prestação de serviços para comunidade data de 1983, "Na área de Relações Públicas foi criada uma Agência Experimental em 1983 (...). As assessorias da área estavam voltadas, até o início da década de 90, para as entidades sem fins lucrativos e possuíam um cunho comunitário ou assistencialista" (Barichello e Martins, 2005, p. 157).

Em 1987 há uma reestruturação curricular no curso de Relações Públicas, as disciplinas Assessoria de Relações Públicas I e II são integradas ao novo currículo. Era o início da organização atual das assessorias. As disciplinas permanecem integrantes da formação obrigatória dos discentes, atualmente, aliam teoria e prática em um trabalho semelhante ao estágio obrigatório existente em outros cursos.

\section{A terceira década}

No ano de 1990 houve uma modificação na estruturação da assistência prestada pelo curso, segundo Barichello e Martins (2005) é implementado um projeto de Assessoria de Comunicação Social, que integrava acadêmicos das três habilitações existentes na época Jornalismo, Publicidade e Propaganda e Relações Públicas. Além do suporte externo de cunho social, passa a ser assistida a direção dos cursos de Comunicação Social. A modificação ainda na segunda década tem suas repercussões na terceira dezena de criação do curso, é por isso que o relato está presente nesta seção.

O projeto vigorou até o ano de 1995 quando "em março de 1995, para dar lugar a um novo projeto, que incluía a instalação de Assessorias de Comunicação nos Centros de Ensino e na Administração Central da Universidade." (Barichello e Martins, 2005, p. 157) cedeu espaço. O programa de extensão "Universidade e Comunidade" teve como premissa o estabelecimento de Assessorias de Relações Púbicas com atuação de acadêmicos e supervisão docente em diferentes órgãos da UFSM. Durante os anos sequentes receberam assessorias os seguintes órgãos/unidades universitárias: Centro de Ciências Rurais; Centro de Ciências Sociais e Humanas; Centro de Tecnologia; Centro de Educação; Centro de Ciências da Saúde; Restaurante Universitário; e Seção Sindical dos Docentes da UFSM. A maior parte destes órgãos ainda mantém assessorias prestadas pelo curso de RP. 
Atualmente, o projeto de extensão "Universidade e Comunidade" está inativo, as assessorias são vinculadas e regidas a partir das disciplinas Assessorias de Relações Públicas I e II ofertadas pelo Curso de Comunicação Social - Habilitação em Relações Públicas, por meio do Departamento de Ciências da Comunicação. Estrutura que vigora até a atualidade. São atendidos sete centros de ensino - Centro de Ciências Sociais e Humanas; Centro de Tecnologia; Centro de Educação; Centro de Ciências da Saúde; Centro de Educação Física e Desportos; Centro de Ciências Naturais e Exatas; Centro de Artes e Letras -, além do Restaurante Universitário e do Hospital Universitário de Santa Maria.

\section{A quarta década}

A quarta década dos cursos de Comunicação Social, sem dúvida, foi a mais agitada: mudanças em ritmo frenético, novidades constante e, sobretudo, grande crescimento, 0 maior de toda a sua história. A criação de um programa de pós-graduação - mestrado e doutorado -; a nova habilitação dos cursos de Comunicação Social - Produção Editorial -; duas reformulações curriculares; aumento do número de vagas para discentes e expansão em infraestrutura e a promessa de um prédio próprio para Comunicação Social; além do registro da maior quantidade e alternância de dirigentes, são os notáveis aspectos nesta década, que estabeleceu de modo singular novos caminhos para os cursos de Comunicação Social da UFSM.

A partir dos anos 2000 as atividades de assessoramento aos órgãos da universidade ganharam maior visibilidade. Soma-se o fomento à produção de relatos científicos e artigos, e também a criação do evento Mostra das Assessorias de Relações Públicas, os quais apresentam à comunidade interessada as atividades de assessoria desenvolvidas pelo Curso.

A Mostra das Assessorias de Relações Públicas - MARP - passou a ser realizada a partir do ano de 2005. A Mostra é vinculada às disciplinas Assessoria de Relações Públicas I e II e possui uma edição em cada encerramento de semestre letivo, sendo que a primeira consiste na exibição dos resultados parciais e planejamentos para o próximo semestre e a segunda na apresentação final com os resultados obtidos durante todo o período da assessoria.

\section{A quinta década}


A nova década, iniciada a partir de 2010, traz novas expectativas para o aprimoramento contínuo das atividades do curso de Relações Públicas. São perspectivas a integração interdisciplinar e o aumento da visibilidade de trabalhos e produções acadêmicas. Atualmente, devido às políticas públicas nacionais na área do ensino superior, como o REUNI $^{3}$, o Curso tem uma média de 120 alunos vinculados e ativos.

O caráter das assessorias de relações públicas como um retorno acadêmico para comunidade, ainda persiste após quase 30 anos da primeira iniciativa, seja através de projetos de extensão, ensino ou pesquisa vinculados às disciplinas Assessoria de Relações Públicas I e II. Estabelecem-se então, além de contribuição social do curso à universidade e comunidade, também como um estágio obrigatório, uma experiência profissional e abertura para o mercado de trabalho local e regional. Trata-se de uma vivência de grande importância proporcionada ao alunado, pois são exigidas inter-relações dos aprendizados das disciplinas anteriores e aplicação dos conhecimentos desenvolvidos.

No ano de 2011 a MARP uniu as disciplinas de Assessoria de Relações Públicas e Cerimonial e Protocolo, transformando-se em uma mostra integrada dos trabalhos realizados, o que obviamente aumentou o público e as trocas de experiências e ideias. Deve ser uma preocupação presente a possibilidade de aumento de dimensões e reconhecimento da Mostra para integrar as atividades do Curso às suas disciplinas, em especial as práticas de assessoria, cerimonial e protocolo e gestão de eventos. Além de estimular a participação de todos os acadêmicos matriculados no Curso. Talvez seja esta uma profícua oportunidade para os discentes compreenderem melhor como, onde, para quê e para quem o profissional de relações públicas trabalha.

Por considerações finais, a reflexão sobre a prática é um imperativo necessário para o desenvolvimento do conhecimento. Estudar a formação do curso de Relações Públicas dimensionada no passado e no presente para assim pensar um futuro pretendido, é um método de buscar o aprimoramento do uso de recursos públicos e do ensino-aprendizagem cidadão para a formação de indivíduos empenhados na construção de um bem-estar coletivo.

O curso de Relações Públicas da Universidade Federal de Santa Maria tem uma carreira acadêmica bastante consolidada. A pesquisa acerca do resgate da memória do Curso se propôs a: compreender a realidade do Curso e complementar estudos da historicidade -

\footnotetext{
${ }^{3}$ Programa de Apoio a Planos de Reestruturação e Expansão das Universidades Federais, mais informações em $<$ http://reuni.mec.gov.br/index.php?option=com_content\&view=article\&id=25\&Itemid=28>.
} 
principalmente da última década -, contribuindo para a reflexão das práticas de ensinoaprendizagem prescritas.

Prognosticamos para o curso um direcionamento para o seu crescimento, principalmente, no âmbito científico. É imprescindível que em conjunto a essa ampliação, também aumente o retorno oferecido à comunidade, o que o Curso já cumpre de modo consolidado há mais de 40 anos em atividades de extensão.

\section{Bibliografía}

Baccin, A. N., Silveira, Santos, C. P., Barichello, E. M. M. R. (1997). Campo profissional e mercados de trabalho em comunicação social dos egressos da FACOS. Anais do III Seminário de avaliação institucional da produção do CCSH. Santa Maria: CCSH, p.61 62.

Barichello, E. M. M. R.; Martins, A. P. (2005) Trajetórias: memórias do curso de comunicação social da UFSM. FACOS-UFSM: Santa Maria.

Barichello, E. M. M. R.; Rhoden, V.; Rosa, R. (2012). Curso de Relações Públicas da UFSM: apontamentos sobre os currículos (1972-2011). Conexão: Comunicação e cultura v.11 n.21.

Curvello, J. J. A. (2002). Comunicação interna e cultura organizacional. São Paulo: Scortecci: São Paulo.

Granez, M. S., Silveira, A. C. M. (1993). A práxis institucional da Faculdade de Comunicação Social e a delimitação do objeto teórico da comunicação. Anais da III Jornada de Pesquisa. Santa Maria: Gráfica Universitária, p. 439.

Ministério da Educação. (2004). INEP. Orientações gerais para o roteiro da auto-avaliação das instituições. INEP: Brasília.

Ministério da Educação. (2008). Instrumento de avaliação institucional externa. INEP: Brasília. 
Universidade Federal de Santa Maria. (1996). Estatuto da UFSM. UFSM: Santa Maria.

Universidade Federal de Santa Maria. (2000). Projeto Político-Pedagógico da UFSM. UFSM: Santa Maria.

Universidade Federal de Santa Maria. (2010). Projeto Político-Pedagógico do Curso de Relações Públicas da UFSM. UFSM: Santa Maria.

\section{Forma de citar este artículo en bibliografías}

Junges Panciera, M.; Ferreira Lisboa Filho, F.; Figueirôa Arruda, C. (2013): "A melhor idade: o curso de Relações Públicas da UFSM aos seus 40 anos", en Revista PANGEA, 4, páginas 136 a 148. Red Académica Iberoamericana de Comunicación. Recuperado el _ de de 2 de: http://www.revistapangea.org 